\title{
On the UWB in-body propagation measurements using pork meat
}

\author{
Mariella Särestöniemi ${ }^{1}$, Carlos Pomalaza-Raez ${ }^{2}$, Chaïmaâ Kissi ${ }^{3}$, Jari Iinatti ${ }^{1}$ \\ ${ }^{1}$ Centre for Wireless Communications, University of Oulu, Finland \\ ${ }^{2}$ Department of Electrical and Computer Engineering, Purdue University, USA \\ ${ }^{3}$ Electronics and Telecommunication Systems Research Group, National School of Applied \\ Sciences (ENSA), Ibn Tofail University, Morocco \\ mariella.sarestoniemi@oulu.fi
}

\begin{abstract}
This paper presents a study on the in-body propagation using pork meat at the lower ultrawideband (UWB) frequency band 3.74-4.25 GHz of the wireless body area network (WBAN) standard 802.15.6. Pork meat in terms of the dielectric properties is one of the most similar to human tissues and thus is commonly used in in-body propagation studies. Nevertheless, there are differences in the dielectric properties, creating some differences also in the radio propagation. The first objective of this paper is to investigate by simulations the propagation differences between human and pork tissue layer models. The simulations results show clear differences between the channel characteristics obtained using a human tissues and pork tissues: within the frequency range of interest, the path loss with pork meat can be up to $5 \mathrm{~dB}$ less than with the human meat. The second objective of this paper is to study, by measurements, the in-body channel characteristics using different types of pork meat piece having different fat and muscle compositions. It was found that path loss is clearly higher with the pork meat having separate skin, fat, and muscle layers compared to the pork meat having interlaced fat and muscle layers. Furthermore, the third objective of this paper is to study the impact of the meat temperature on the measured channel characteristics by comparing the channels obtained with the meat at the temperatures of $12^{\circ} \mathrm{C}$ and at $37^{\circ} \mathrm{C}$. Also, in this case clear differences were observed in path loss: within the frequency range of interest, the path loss was maximum 5 $\mathrm{dB}$ lower with meat at $37^{\circ} \mathrm{C}$ than with a colder meat. The results presented in this paper provide useful information and relevant aspects for the in-body propagation studies conducted with pork meat.
\end{abstract}

Keywords: dielectric properties of tissues, fat layer propagation, in-body propagation, radio channel measurements, temperature impact, ultra wideband, wireless body area networks.

\section{$1 \quad$ Introduction}

Wireless medical monitoring has increased interest in recent years due to the several benefits it may provide for the healthcare of human beings [1]-[3]. Capsule endoscope 
is one example of the implant monitoring systems which has become popular method of investigating the gastrointestinal (GI) tract [4]-[5].

Smooth design of monitoring devices requires deep knowledge of radio channel characteristics. Channel characteristics can be studied with electromagnetic simulations, which are based on solving Maxwell's equations in their differential or integral forms, or measurements using phantom models, anaesthetized living animals, or tissues of the animals [5]-[8].

Measurements with the anaesthetized animals provide the most realistic results of the measurements since dielectric properties of the tissues start to change immediately after the death of the animal [9]. However, measurements are challenging with anaesthetized animals since such measurements require hospital environment [10]-[11]. Instead, measurements with meat pieces are more feasible. Since some of the tissue dielectric properties of adult pig is known to be close to the those off human beings, pig meat is commonly used in the on-body/in-body antenna studies as well as in in-body propagation studies [12]-[14].

However, the use of meat pieces has some shortcomings and restrictions which should be considered. For instance, dielectric properties of animals can be different compared to those of human beings though dielectric properties of adult pork tissues are found to be close to those of humans [15]-[17]. Besides, dielectric properties of tissues change with the age of the animal [18] or as the time passes from the death of the animal [9]. Moreover, temperature impacts the dielectric properties of the tissues [16], which obviously has clear impact on the in-body channel characteristics. Finally, composition of fat and muscle layers in the meat piece is assumed to have strong impact on the results since fat is known to be a good propagation channel [19]-[20].

Up to the author's knowledge, there is no studies presented on comparing channel characteristics evaluated with meat pieces having different fat and muscle composition with realistic antennas. Furthermore, up to the author's knowledge there are no studies presenting impact of the meat temperature on the channel characteristics.

The aim of this paper is to address the aforementioned aspects. First, channel characteristics obtained using human tissues and pork tissues in the layer simulation models are compared. Secondly, the channel characteristics are evaluated using two different meat pieces: the first one having interlaced muscle and fat layers, the second one having separate muscle and fat layers. Finally, the impact of the meat's temperature is evaluated on the antenna performance and channel characteristics.

The paper is organized as follows: Section II presents study case including description of the simulations, antennas, and measurements. Section III presents simulation based antenna verification. Section IV compares channel characteristics obtained using human tissues and pork tissues in the layer simulation model. Measurement results for meat pieces with different fat and muscle composition are evaluated in Section IV. Furthermore, the impact of the temperature on the antenna and channel characteristics are studied in Section IV. Finally, Conclusions with future work perspectives are discussed in Section V. 


\section{Study Case}

\subsection{Simulations}

Simulations were conducted with a 3D electromagnetic simulation tool CST Studio Suite software [21], which uses the finite integration technique. A planar layer tissue model, which is presented in Fig. 1, was used in the simulations. The thicknesses of the tissue layers are selected according to the thicknesses of tissue layers of the meat piece 1 used in the measurements. These thicknesses are presented in Table I in Subsection 2.2

In this study, we use two types of antennas. The first one is a directional cavity backed UWB on-body antenna, shown in Fig. 2a, which was introduced in [22]. It is designed for on-in body communications for the frequency band 3.75-4.25 GHz which meets the IEEE 802.15.6 standards requirements [23]. Its dimensions with the cavity are $x=83 \mathrm{~mm}$, $\mathrm{y}=49.5 \mathrm{~mm}$, and $\mathrm{z}=19.62 \mathrm{~mm}$, where $\mathrm{x}$ and $\mathrm{y}$ are width and length, respectively and $\mathrm{z}$ is towards the body. The size of the antenna itself is $x=47.5 \mathrm{~mm}, y=47.5 \mathrm{~mm}$. The second antenna is a loop antenna, shown in Fig. 2b, which is introduced in [24]. The loop antenna is omni-directional antenna UWB antenna, having large bandwidth 3.1-10.6 GHz and it is originally designed for on-body communications. The size of the antenna is $43 \mathrm{~mm} x$ $46 \mathrm{~mm}$. In this initial study, the loop antenna is used to resemble the in-body antenna, since in the simulations, it was noticed to achieve similar path loss values as the implant (capsule endoscope) antenna [25], for which a prototype was not available.

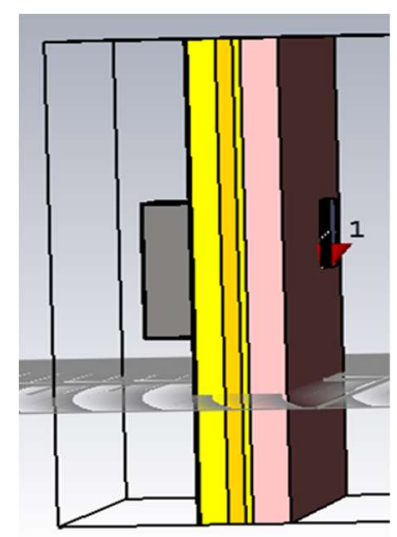

Fig. 1. Layer model used for the verification of the antennas. 
a)

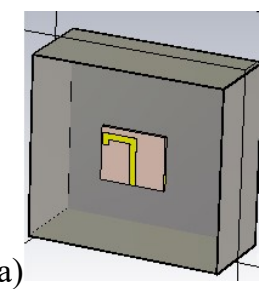

b)

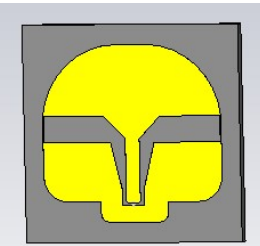

Fig. 2. Antennas used in this study: a) cavity backed low-UWB on-body antenna, b) loop antenna.

\subsection{Measurements}

Measurements were conducted using two different types of porcine pieces which are presented in Figs. 3a-b. Both pieces had skin, outer fat, muscle and inner fat layers. In the first meat piece, which is referred to as Meat 1 , the fat and muscle layers are interlaced so that there are "fat tunnels" going through the muscle layer. Meat 2 has distinct fat and muscle layers. Both meat pieces have thickness of $3 \mathrm{~cm}$. The thickness of the tissues layers for Meat 1 and Meat 2 below the antennas are presented in Table I.

The measurements were conducted with a Vector Network Analyzer (VNA) 8720ES in measurement laboratory premises at the University of Oulu, Finland. The meat pieces were set individually inside a cube form area made by absorber pieces to avoid signal interference from the surrounding environment as well as minimize the propagation overflow from the sides of meat pieces. Meat piece was set inside a thin plastic bag to protect the antennas and the absorbers. The picture of the measurement setup is presented in Fig. 4.

a)

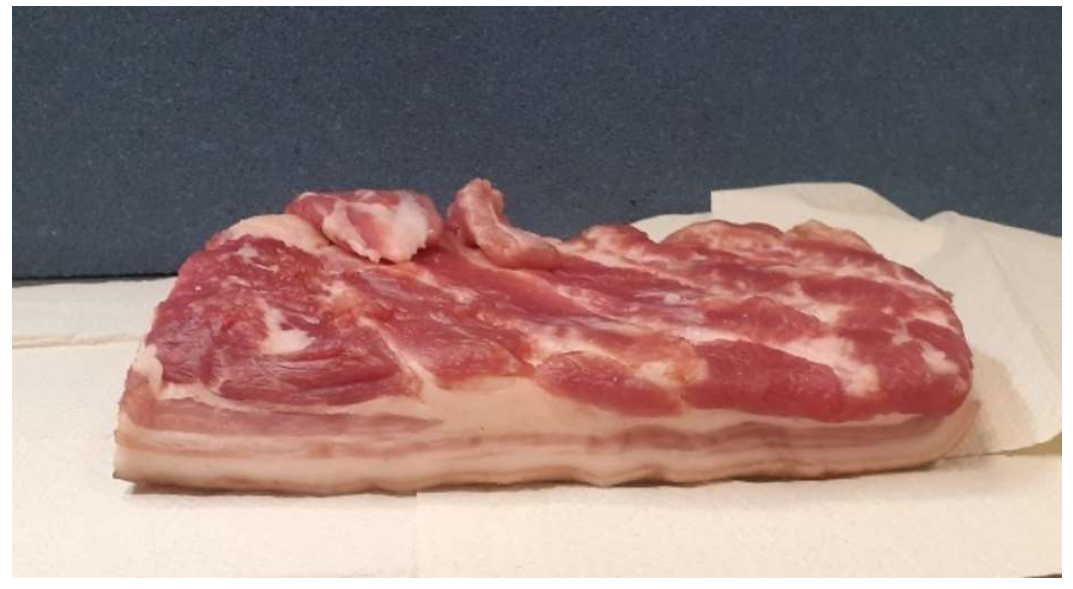


b)

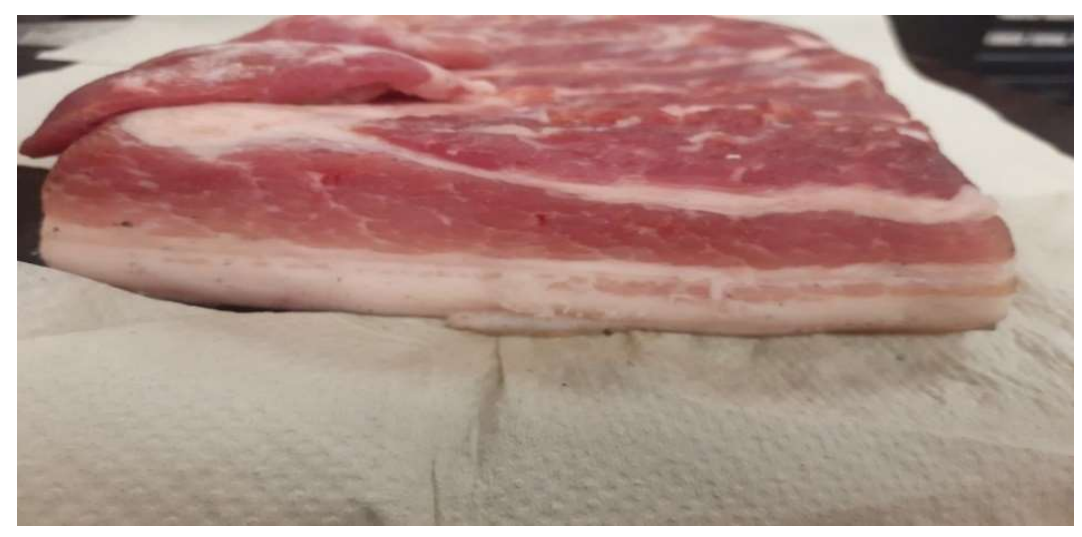

Fig. 3. a) Meat 1 with interlaced fat and muscle layers, b) Meat 2 with distinct muscle and fat layers.

Table I. Tissue thicknesses of Meat 1 and Meat 2 in the location of antennas.

\begin{tabular}{lll}
\hline Tissues & Meat1 & Meat2 \\
\hline Skin & $2 \mathrm{~mm}$ & $2 \mathrm{~mm}$ \\
Outer fat & $10 \mathrm{~mm}$ & $7 \mathrm{~mm}$ \\
Muscle & $15 \mathrm{~mm}$ & $18 \mathrm{~mm}$ \\
Inner fat & $3 \mathrm{~mm}$ & $3 \mathrm{~mm}$ \\
\hline
\end{tabular}
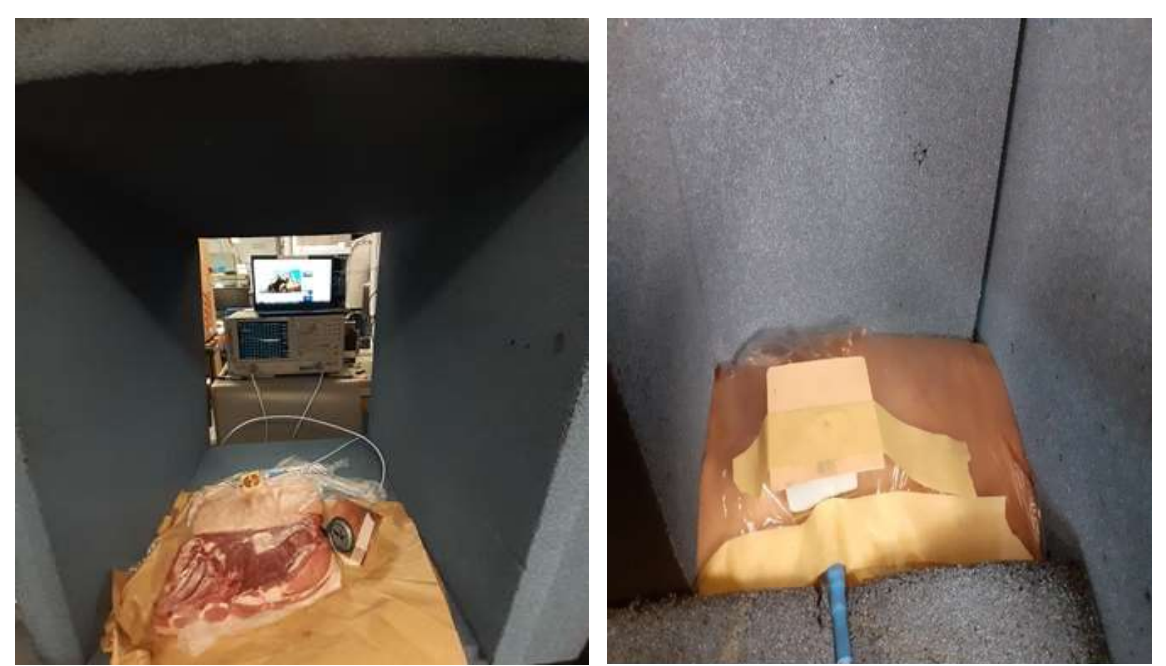

Fig. 4. a) Measurement setup before closing the back and front walls, b) on-body antenna with meat piece 


\section{Simulation based antenna verification}

The aim of this section is to verify usability of the loop antenna on the measurement based in-body propagation studies. This verification is conducted by comparing simulated channel characteristics obtained using an implant antenna [25] and a loop antenna [24] as the in-body antenna. The simulations are conducted using the planar tissue layer model since the pork meat piece also resembles planar layer model. The aim of this comparison is to show that loop antenna can be considered as an alternative in the measurements when using layer meat pieces. Fig. 5 presents comparison between the frequency and time domain channel characteristics obtained using a capsule antenna and the loop antenna in the simulations. Fig. 5a presents the S21 for the whole simulated frequency range and Fig. 5b the zoomed version of Fig. 5a for the frequency band of interest 3.75-4.25 GHz. Fig. 5c presents channel impulse responses obtained applying inverse fast Fourier transform (IFFT) for the simulated band. As one can note from Figs. 5a-b, the path loss difference is minor between the results obtained using the capsule antenna and the loop antenna within frequency range of interest. However, outside the frequency range of interest the differences are larger.

In time domain, the main peaks of the IRs are at the same level, though the main peak is clearly wider with the loop antenna. From the results it can be concluded that loop antenna can be used as in-body propagation studies in the measurements.

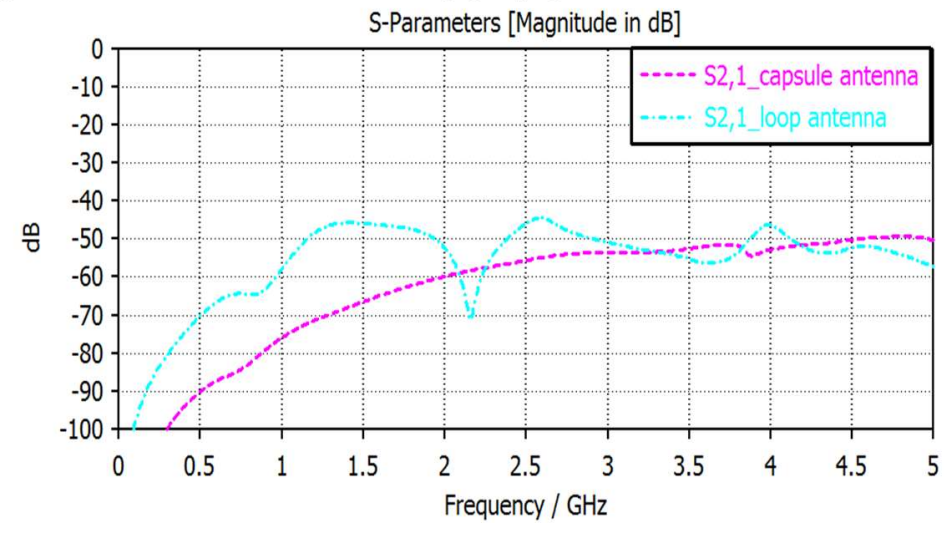

a) 


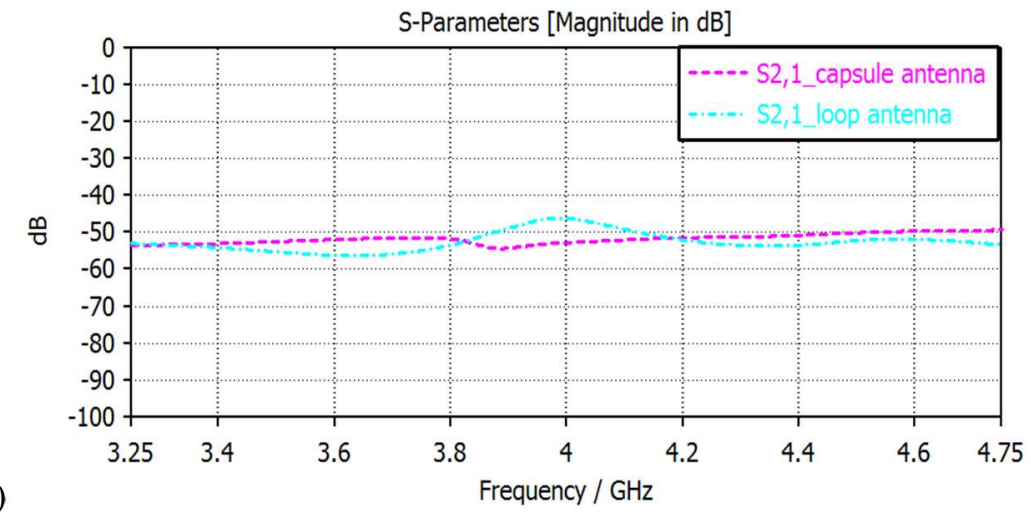

b)

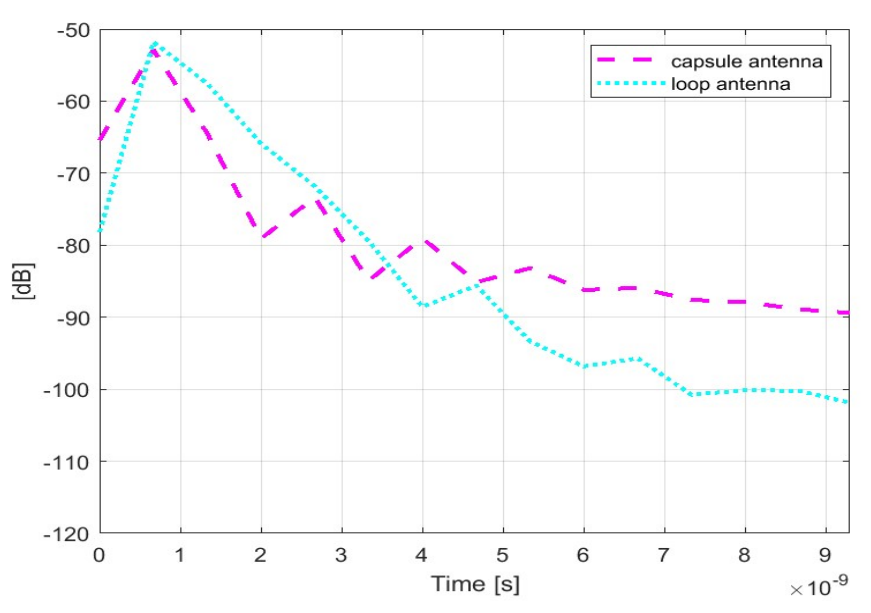

Fig. 5. Simulation based comparison of channel characteristics obtained using a loop antenna and a capsule endoscope implant antenna [25]: a) S21s for the whole simulated bandwidth, b) S21 for the frequency range of interest, and c) impulse responses.

\section{Simulation based comparison between the channel characteristics obtained using human and pork tissues}

This section compares simulation results obtained using tissue layer models having dielectric properties of human tissues to those obtained using pork tissues. Dielectric properties (relative permittivity and tangent loss) for human and pork tissues at $4 \mathrm{GHz}$ are presented in Table II. The dielectric properties of human tissues are found from e.g. [26]. For dielectric properties of pork tissues there are several studies available in the literature [9]-[10], [15]-[16]. However, the challenge is there is variation in the values presented for pork. The reason for this is that several factors affect the dielectric properties: age and size of the pig, time from the death of the pig, temperature of the meat. For instance, there is wide variation in the reported results for tangent loss values of the 
pork muscle tissue. Thus, for this study, we decided to use tangent loss value of the human tissues also for pork tissues and we left study of the impact of the reported tangent loss variation for future work.

Table II. Dielectric properties of human and pork tissues at $4 \mathrm{GHz}$.

\begin{tabular}{lll}
\hline Tissues & $\begin{array}{l}\text { Human tissues } \\
\text { Relative permittivity/ tangent loss }\end{array}$ & $\begin{array}{l}\text { Pork tissues } \\
\text { Relative permittivity }\end{array}$ \\
\hline Skin & $36.6 / 0.29$ & $37.5 / 0.32$ \\
Fat & $5.12 / 0.16$ & $5.78 / 0.23$ \\
Muscle & $50.8 / 0.27$ & $48.0 / 0.27$ \\
\hline
\end{tabular}

First, the antenna matching with human and pork tissues are evaluated by studying the S11 parameters presented in Figs. 6a-b for the on-body antenna and the loop antenna, respectively. It is found that the difference between the dielectric properties of human and pork tissues cause approximately $3 \mathrm{~dB}$ difference in the antenna's notch area so that $\mathrm{S} 11$ values is slightly higher with the human tissues. Same phenomenon can be found with both antennas.

The frequency and time domain channel characteristics are presented in Figs. $7 \mathrm{a}-\mathrm{b}$, respectively. As it is noted, difference in the dielectric properties of human and pork tissues have clear impact on the channel characteristics: within the simulated bandwidth, the path loss is up to $8 \mathrm{~dB}$ higher with pork tissues until $3.25 \mathrm{GHz}$ than with the human tissues. Instead, from $3.5 \mathrm{GHz}$ onwards, i.e. also within the frequency range of interest 3.75 - 4. $25 \mathrm{GHz}$, the path loss is higher with human tissues.

In time domain, as the IFFT is performed for the whole simulated bandwidth, the difference can be seen mainly in within the time range of 1-2 ns, where the level of the channel taps is higher with the human tissues than with the pork tissues. However, as it was pointed out that S21 is higher with pork tissues at the frequency range of interest, it is important to study impulse response also with the filtered S21. The IR obtained by performing IFFT only for the frequency range $3.75-4.25 \mathrm{GHz}$, is presented in Fig. 7c. In this case, it can be clearly seen that the IR peaks are 1-3 dB stronger with pork tissues. Even the difference in the main peaks in almost $3 \mathrm{~dB}$. 


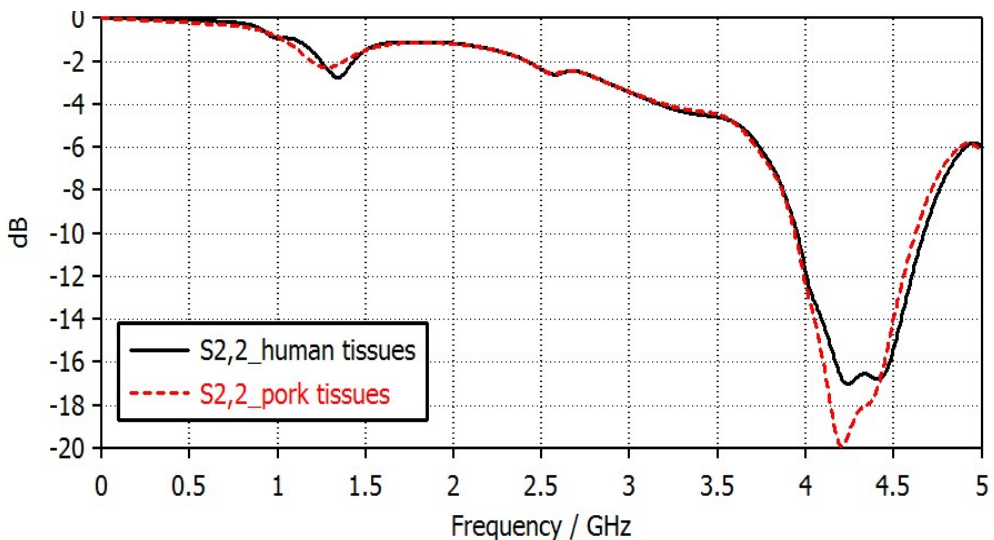

a)

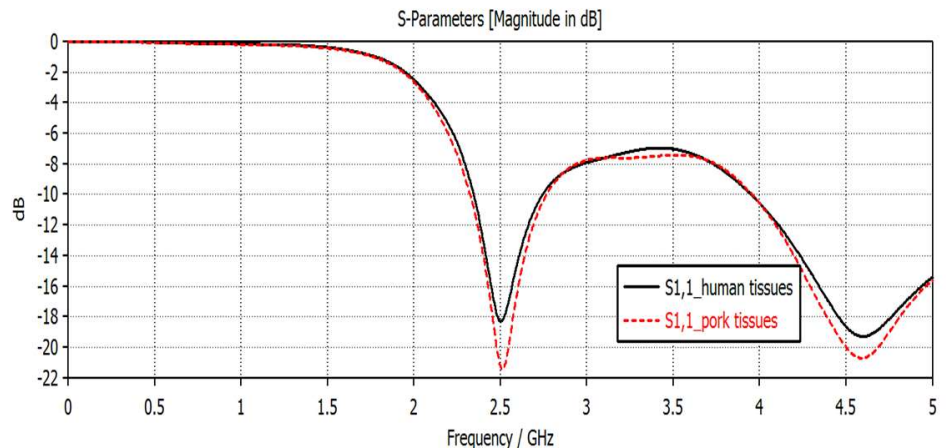

b)

Fig. 6. S11 parameters with a) on-body antenna, b) loop antenna (in-body antenna) obtained with simulation model having dielectric properties of human tissues and pork tissues.

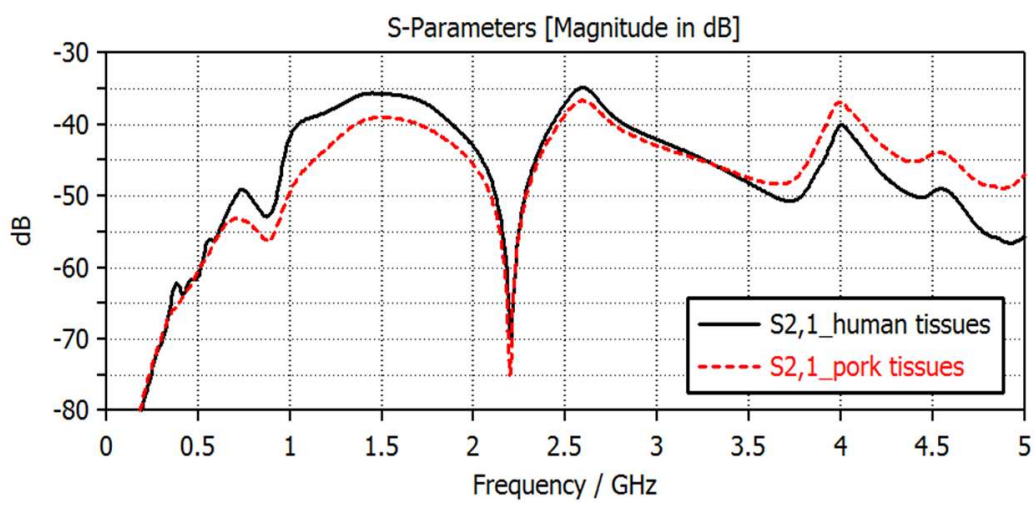

a) 


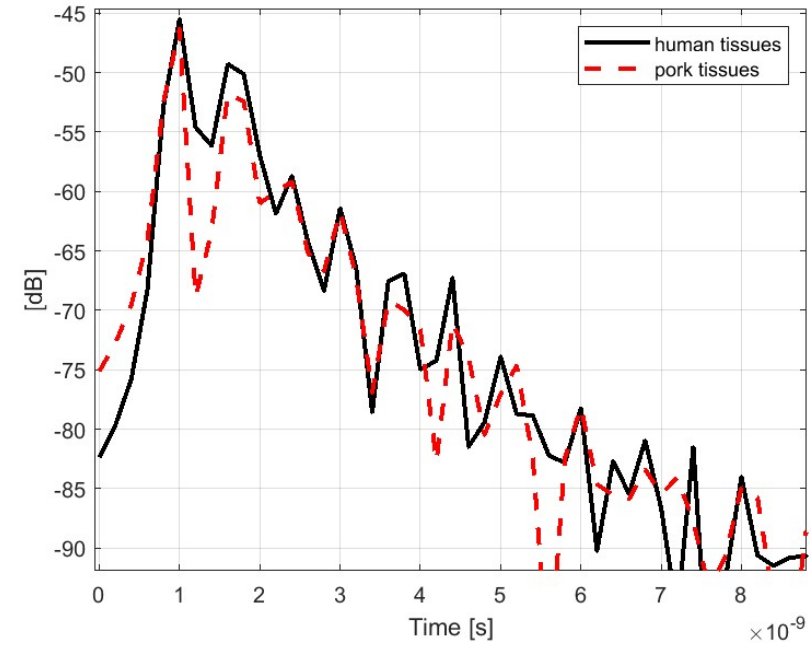

b)

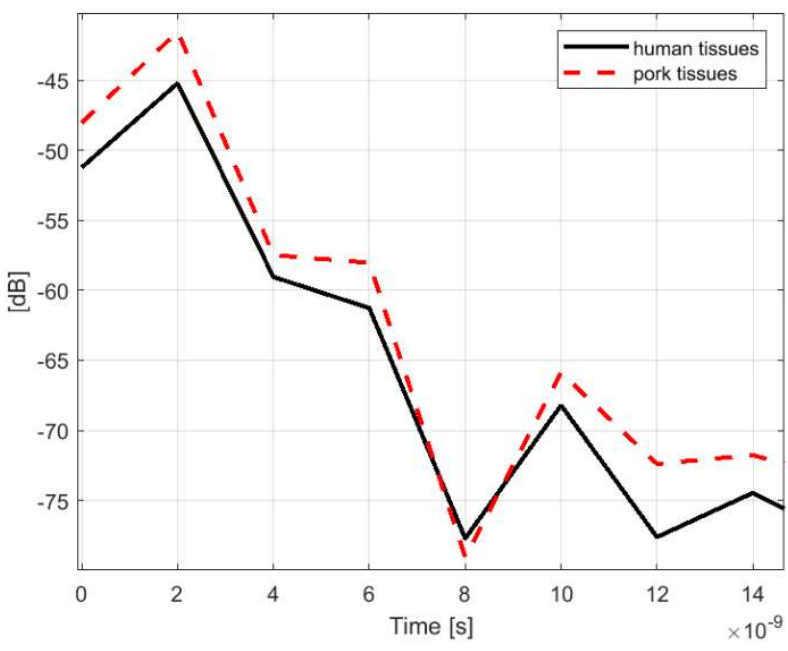

c)

Fig. 7. Comparison between the differences of human and pork tissues: a) S21 parameters, b) IRs obtained by performing IFFT for whole the frequency bandwidth, and c) IRs obtained by performing IFFT for whole the frequency band of interest $3.75-4.25 \mathrm{GHz}$.

\section{$5 \quad$ Measurement results}

\subsection{Channel comparison with different meat pieces}

In this section, the frequency and time domain channel characteristics are evaluated and compared using Meat1 and Meat2, which both have thickness of $3 \mathrm{~cm}$. The aim is to observe how the meat constitution, i.e. whether the fat and muscle layers are interlaced or separated, have impact on the channel characteristics. 
Firstly, the antenna reflection coefficients are on-body and the loop antenna are studied when located on or below the pork pieces Meat1 and Meat2. The reflection coefficients for the on-body antenna is presented in Fig. 8a and for the loop antenna in Fig. 8b. Antenna reflection coefficients for the on-body antenna are very similar with both meat pieces, since the skin thickness and outer fat thickness are same with both meats. Instead, one can note differences in the reflection coefficients of the loop antenna, which is located below the meat pieces. This is due to the different tissue constitution of these different pork meats: there is more fat on the inner surface of Meatl whereas more muscle tissues on the inner surface of Meat2. The differences in the antenna matching are noteworthy only at $1.5 \mathrm{GHz}$ and $2.5 \mathrm{GHz}$, which however, are out of the frequency range of interest. The antenna matching at $3.75-4.25 \mathrm{GHz}$ is relatively same with both meat pieces.

a)
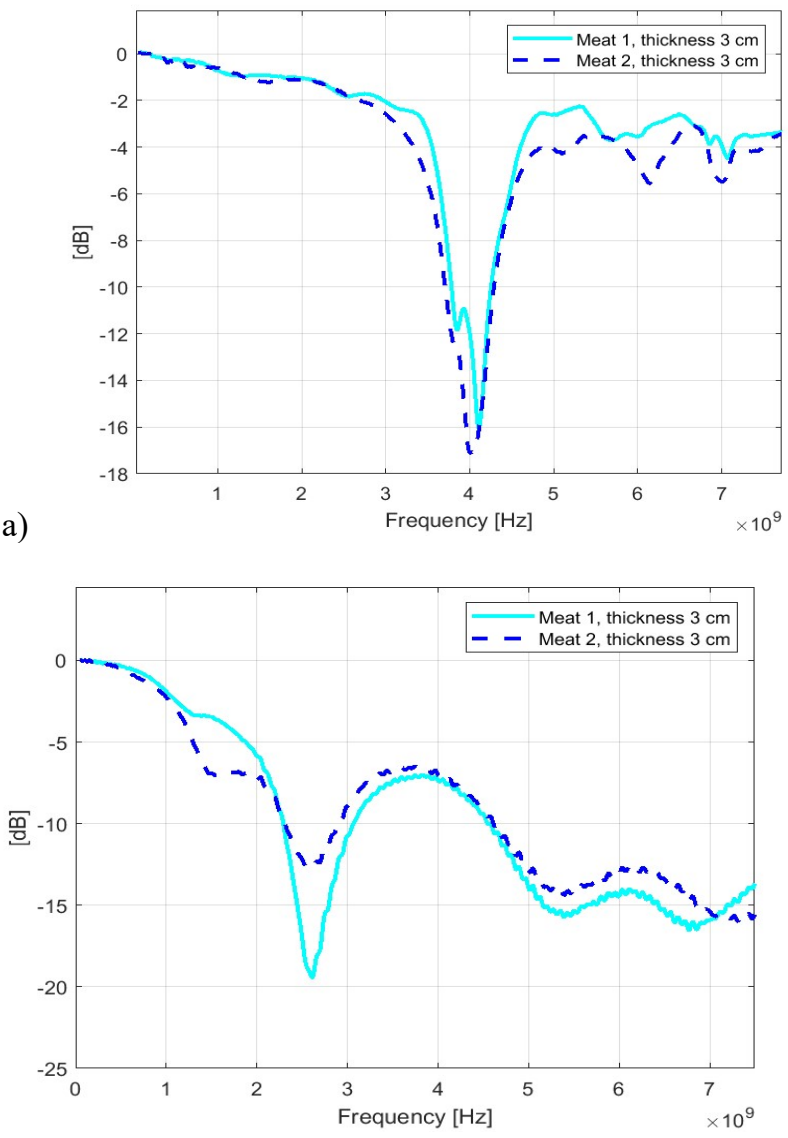

b)

Fig. 8. Reflection coefficient for a) on-body antenna, b) the loop antenna within the tissues with Meat1 and Meat2. 
Next, the channel characteristics are studied. Frequency domain channel characteristics, i.e. S21 parameters, and time domain channel characteristics, i.e. channel impulse responses, are presented in Figs. 9a-b. As one can note, there is a clear level difference in the frequency and time domain channel characteristics obtained using Meat 1 and Meat 2 in the measurements. In frequency domain, the level difference is clearest at 1.5 $\mathrm{GHz}$ and $3 \mathrm{GHz}$ which, however, are out of the frequency range of interest. At the onbody antenna's operational frequency range, 3.75-4.25 GHz, the level difference is at highest $10 \mathrm{~dB}$, except at $3.75 \mathrm{GHz}$ there is a large notch with Meat1. In time domain, the main difference is observed in the level and width of the main peak. The difference is also in this case at highest $10 \mathrm{~dB}$.

The differences in the channel characteristics obtained using Meat1 and Meat2 are partly explained by differences in the antenna matching. However, the main difference comes from the different propagation paths due to different tissue composition. Fat is known to be one of the "easiest" tissue for UWB signal propagation in terms of propagation time and power loss [19]-[20], hence, Meat1 having "fat tunnels" through the muscle layer appear to be clearly easier meat piece to propagate through.

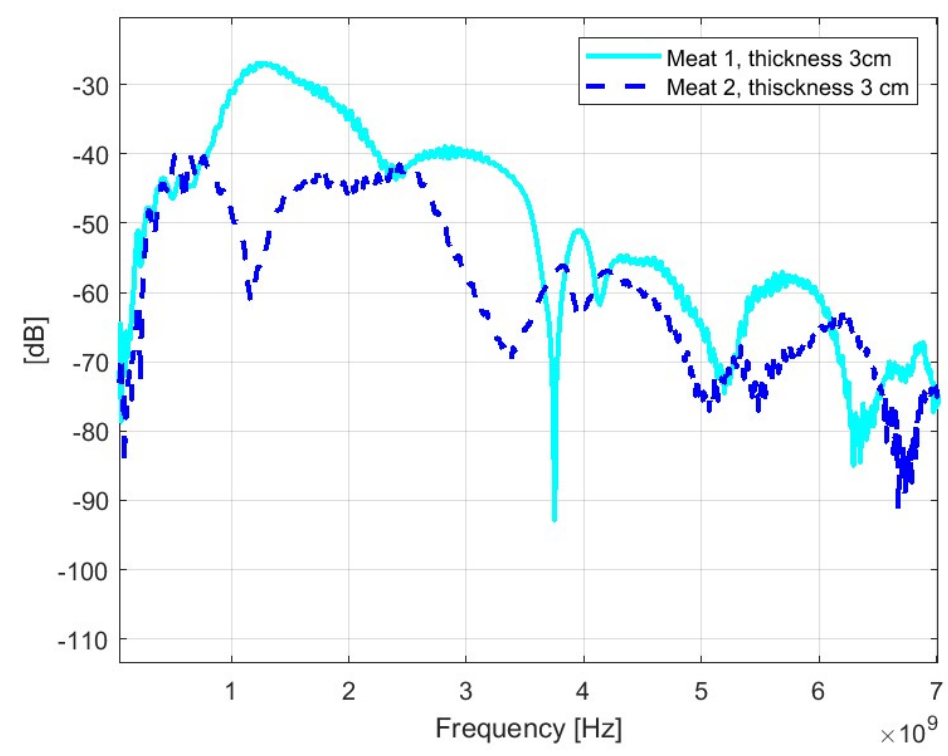

a) 


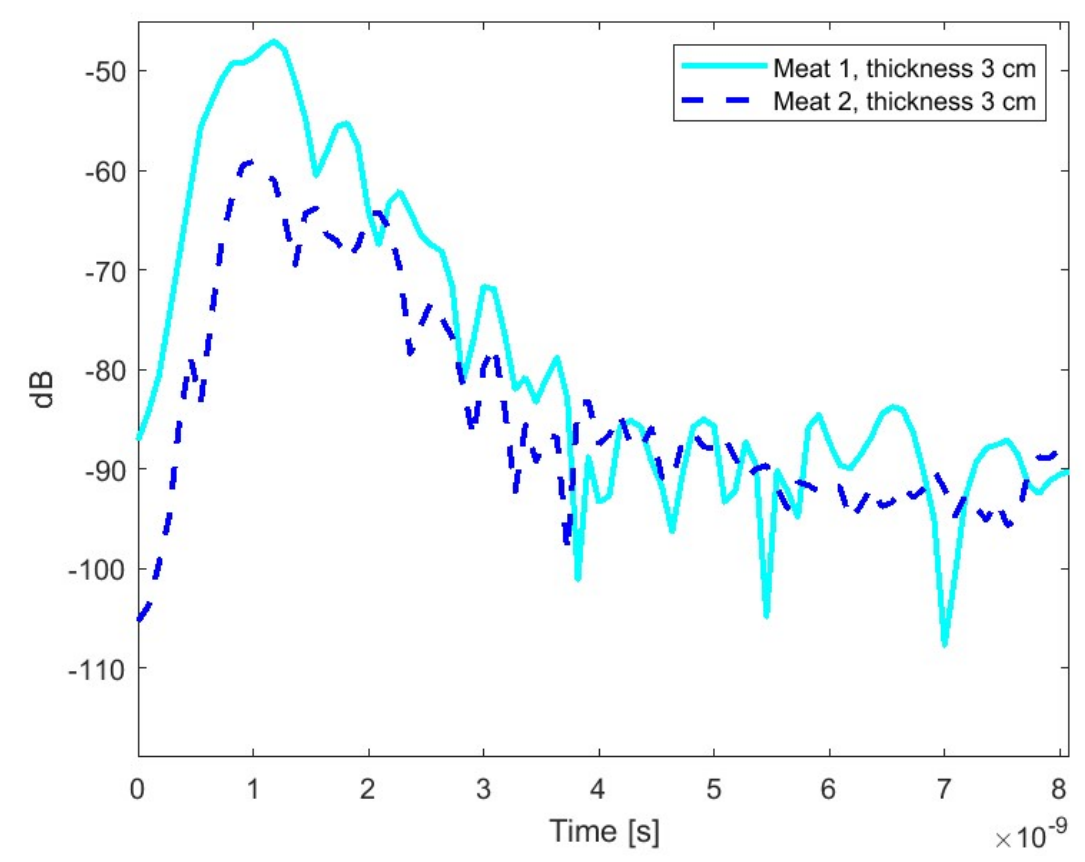

b)

Fig. 9. a) Frequency domain and b) time domain comparison of channel characteristics obtained using Meat 1 and Meat2.

\section{$6 \quad$ Impact of the temperature}

The channel results presented in the previous section were evaluated for the meat pieces which was measured to have temperature of $12^{\circ} \mathrm{C}$. However, temperature of the humans is approximately $37^{\circ} \mathrm{C}$. The temperature is known to have a clear impact on the tissues' dielectric properties [16], which is assumed to change also channel characteristics significantly. Thus, next, the impact of the temperature is evaluated for channel characteristics with Meatl.

The Meat 1 was heated in a heating box up to $37^{\circ} \mathrm{C}$. Then the S11, S21, and IR values are evaluated in the same antenna positions as in Section A. The obtained results are presented in Figs. 10a-c. For the comparison, S11, S21, and IR values measured in the cold $\left(12^{\circ} \mathrm{C}\right)$ temperature are included in the figure.

As noted from Fig. 10a, temperature changes the antenna matching slightly at the lower frequencies (up to $4.1 \mathrm{GHz}$ ). Due to these changes, the notch of the $\mathrm{S} 11$ is transferred $0.2 \mathrm{~dB}$ to the lower frequency, to $3.9 \mathrm{GHz}$. The changes in the $\mathrm{S} 21$ values are also noteworthy at lower frequencies, as presented in Fig. 10b. However, at the frequency range of interest, the difference is maximum $5 \mathrm{~dB}$. In time domain results, 
presented in Fig. 10c, the main difference can be noted in the level of the CIR's main peak: with warm meat, the main peak is $9 \mathrm{~dB}$ at higher level than that with the cold meat.

a)

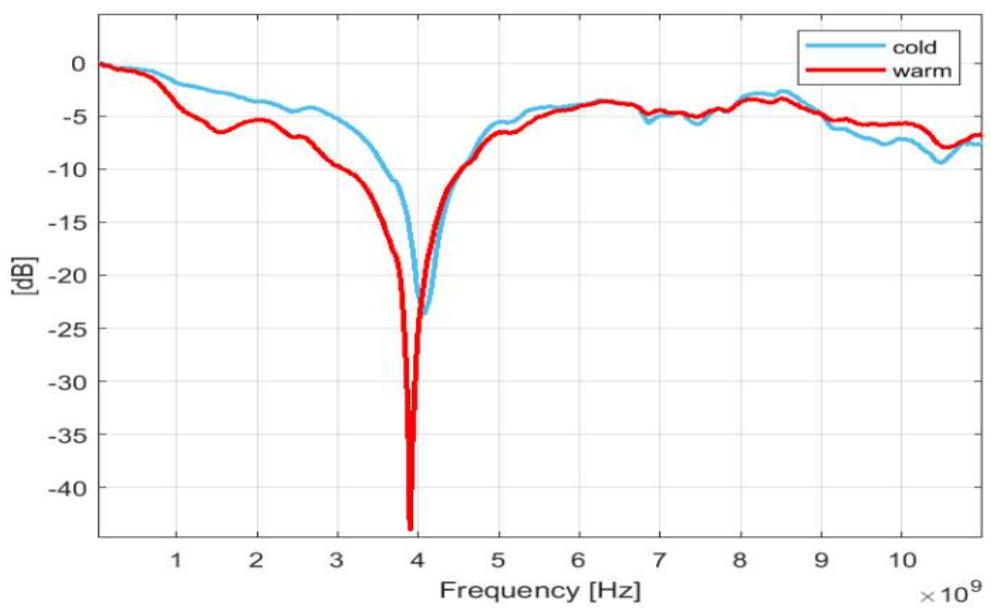

b)

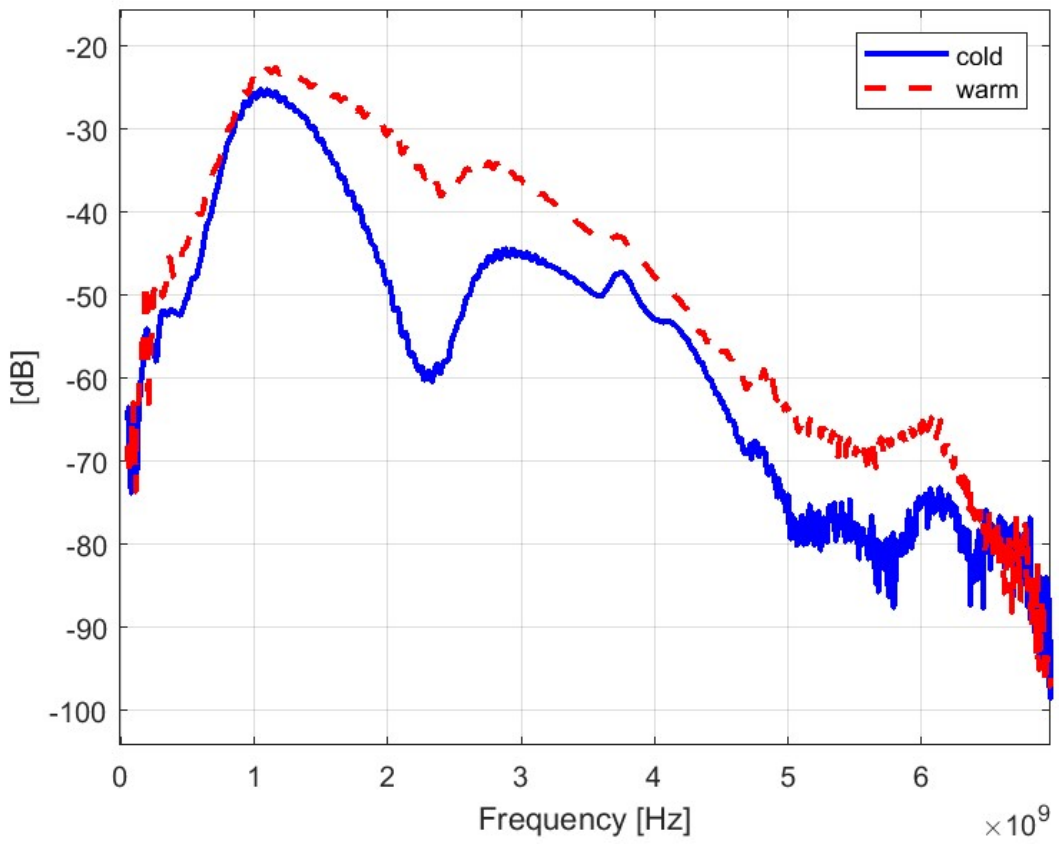


c)

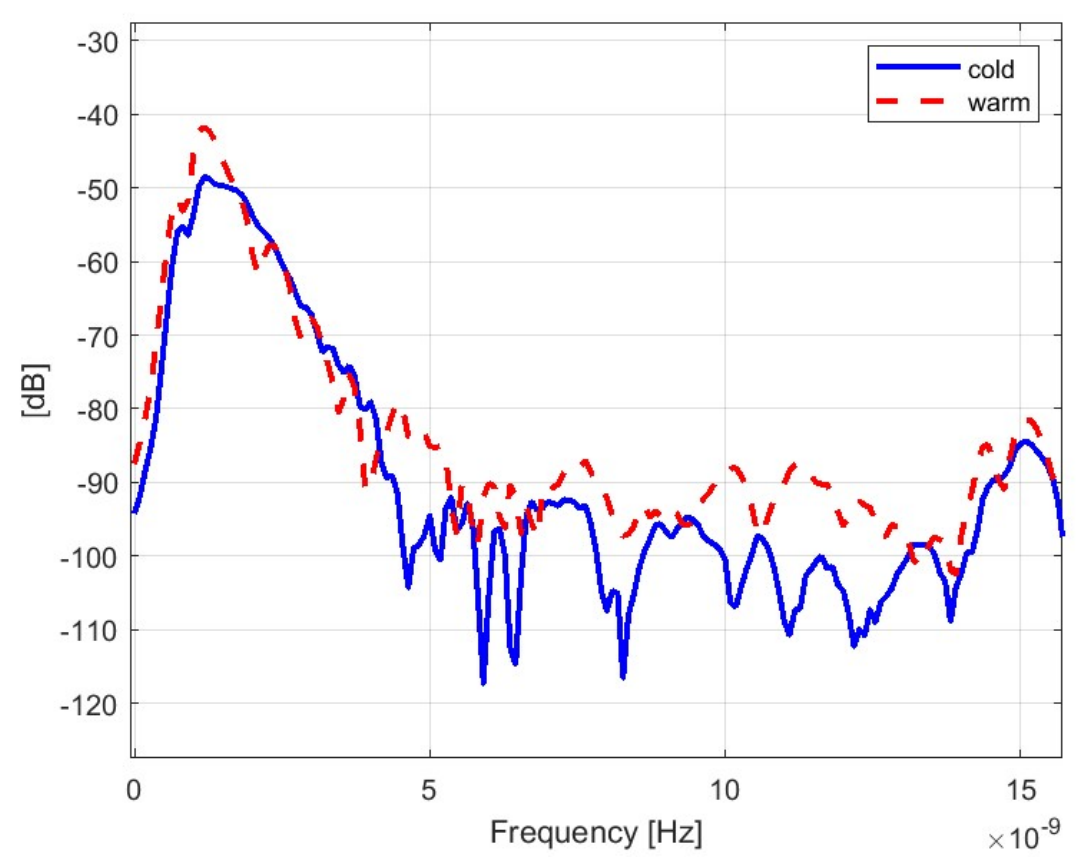

Fig. 20. Comparison between the antenna and channel characteristics at $12^{\circ} \mathrm{C}$ and $37^{\circ} \mathrm{C}$ temperature: a) S11 parameters for on-body antenna, b) S21 parameters, and c) IRs obtained performing IFFT for the whole frequency bandwidth.

\section{Conclusions}

This paper presented a study on UWB in-body propagation measurements conducted using pork meat. The first aim of this paper was to investigate by simulations the propagation differences between human and pork tissue layer models. The simulations results showed clear differences between the channel characteristics obtained using human tissues compared to pork tissues: within the frequency range of interest at $3.75-$ $4.25 \mathrm{GHz}$, the path loss difference is up to $5 \mathrm{~dB}$. The second aim of this paper was to study, by measurements, the propagation using different types of pork meat piece having different fat and muscle compositions. It was found that path loss is clearly higher with the pork meat having separate skin, fat, and muscle layers compared to the pork meat having interlaced fat and muscle layers. Furthermore, the third objective of this paper was to study the impact of the meat temperature on the measured channel characteristics by comparing the channels obtained with the meat at the temperature of $12^{\circ} \mathrm{C}$ and at $37^{\circ} \mathrm{C}$. Also, in this case clear differences were observed in path loss: within the frequency range of interest, the path loss was maximum $5 \mathrm{~dB}$ lower with meat at $37^{\circ} \mathrm{C}$ than with a colder meat. 
The results presented in this paper provide information and insights on the use of pork meats in the in-body propagation studies. It is shown that selection of the meat pieces used in the measurements is crucial: meat composition may have strong impact on the channel characteristics. Besides, it would be important to heat the meat up to $37^{\circ} \mathrm{C}$ if more realistic scenario is aim to be evaluated. Furthermore, although the dielectric properties of the human and adult pork tissue are similar, the differences in the dielectric properties cause clear impact on the channel characteristics, which should be taken into the account in monitoring device design.

Our future work plans include extensive measurements using different meat pieces having different thicknesses with different fat and muscle composition. Aim is to perform propagation path calculations for different propagation path options with the corresponding dimensions and compare them with the measured data. Furthermore, different on-body and implant antennas having different operational frequencies will be used in the evaluations.

\section{Acknowledgement}

This work is supported in part by the projects WBAN Communications in the Congested Environments (MeCCE), the Academy of Finland 6Genesis Flagship (grant 318927) and the European Union's Horizon 2020 programme under the Marie Sklodowska-Curie grant agreement No. 872752. Dr. Marko Sonkki is acknowledged for his participation on the on-body antenna design.

\section{References}

1. R. K. Pathinarupothi, P. Durga, E. S. Rangan, "IoT Based Smart Edge for Global Health: Remote Monitoring with Severity Detection and Alerts Transmission," IEEE Internet of Things Journal, Eraly Access 2019.

2. E. Schires, P. Georgiou, T. S. Lande, "Vital Sign Monitoring Through Back Using an UWB Impulse Radar with Body Coupled Antennas," IEEE TRANSaction on Biomedical Circuits and Systems, 2018.

3. P. Leelatien, K. Ito, K. Saito, M. Sharma, A. Alomainy, "Channel Characteristics and Wireless Telemetry Performance of Transplanmted Organ Monitoring System Using Ultrawideband Communication," IEEE Journal of Electromagnetics, RF and Microwaves in Medicine and Biology, 2018.

4. G. Ciuti, A. Menciassi, and P. Dario, "Capsule endoscopy: from current achievements to open challenges," IEEE Reviews in Biomedical Engineering, vol. 4, pp. 59-72, 2011.

5. O. Bchir, M. M. ben Ismail, and N. AlZahrani, "Multiple bleeding detection in wireless capsule endoscopy," Signal, Image and Video Processing, vol. 13, no. 1, pp. 121-126, 2018.

6. Ali Fatih Demir, Qammer H. Abbasi, Z. Esat Ankarali, Akram Alomainy, Khalid Qaraqe, Erchin Serpedin, Huseyin Arslan, "Anatomical Region-Specific In Vivo Wireless Communication Channel Characterization", Biomedical and Health Informatics IEEE Journal of, vol. 21 , no. 5, pp. 1254-1262, 2017 
7. C. Garcia-Pardo, A. Fornes-Leal, N. Cardona, R. Chávez-Santiago, J. Bergsland, I. Balasingham, S. Brovoll, Ø. Aardal, S.-E. Hamran, R. Palomar, "Experimental ultra wideband path loss models for implant communications", Personal Indoor and Mobile Radio Communications (PIMRC) 2016 IEEE 27th Annual International Symposium on, pp. 1-6, 2016

8. Carlos Andreu, Sergio Castelló-Palacios, Concepcion Garcia-Pardo, Alejandro Fornes-Leal, Ana Vallés-Lluch, Narcís Cardona, "Spatial In-Body Channel Characterization Using an Accurate UWB Phantom", Microwave Theory and Techniques IEEE Transactions on, vol. 64, no. 11 , pp. 3995-4002, 2016

9. A. Surowiec, S. Stuchly, A. Swarup, "Radiofrequency dielectric properties of animal tissues as a function of time following death", Physics in Medicine \& Biology, Vol. 30, Nro 10, Oct. 1985.

10. T. Karacolak, R. Cooper, E. S. Unlu and E. Topsakal, "Dielectric properties of porcine skin tissue and in vivo testing of implantable antennas using pigs as model animals", IEEE Antennas and Wireless Propagation Letters, vol. 11, pp. 1686-1689, 2012

11. Yuto Shimizu, Daisuke Anzai, Raul Chavez-Santiago, Pål Anders Floor, Ilangko Balasingham, Jianqing Wang, "Performance Evaluation of an Ultra-Wideband Transmit Diversity in a Living Animal Experiment", Microwave Theory and Techniques IEEE Transactions on, vol. 65, no. 7, pp. 2596-2606, 2017.

12. P. A. Floor et al., "In-Body to On-Body Ultrawideband Propagation Model Derived From Measurements in Living Animals," in IEEE Journal of Biomedical and Health Informatics, vol. 19, no. 3, pp. 938-948, May 2015, doi: 10.1109/JBHI.2015.2417805.

13. K. M. S. Thotahewa, J. Redoutè and M. R. Yuce, "Propagation, Power Absorption, and Temperature Analysis of UWB Wireless Capsule Endoscopy Devices Operating in the Human Body," in IEEE Transactions on Microwave Theory and Techniques, vol. 63, no. 11, pp. 3823-3833, Nov. 2015, doi: 10.1109/TMTT.2015.2482492.

14. O. Novak and R. B. Brown, "An empirical model of UWB large-scale signal fading in neocortical research," 2016 IEEE International Symposium on Circuits and Systems (ISCAS), Montreal, QC, 2016, pp. 2439-2442, doi: 10.1109/ISCAS.2016.7539085.

15. C. Gabriel, S. Gabriel, E. Corthout, "The dielectric properties of biological tissues: I. Literature Survey," Physics in Medicine and biology, 41 (1996), 2231-2249.

16. S. Ley, S. Schilling, O. Fiser, J. Vrba, J. Sachs, M. Helbig, "Ultra-wideband temperature dependent dielectric spectroscopy of Porcine tissue and blood in the microwave frequency"

17. A. Peyman, C. Gabriel, "Cole-cole parameters for dielectric properties of porcine tissues as a function of age at microwave frequencies," Physics in Medicine and biology, 55, N 413, 2010.

18. M. Ngadi, S. Dev, V. G.S. Raghavan, S. Kazemi, "Dielectric properties of pork muscle," International Journal of Food Properties, Sept. 2015.

19. M. Särestöniemi, C. Pomalaza-Raez, M. Berg, C. Kissi, M. Hämäläinen. J. Iinatti, "Fat in the Abdomen as a Propagation Medium in WBAN Applications," Bodynets2019.

20. N. B. Asan, E. Hassan, J. Shah, D. Noreland, T. Blokhuis, R. Augustine, "Characterization of the fat channel for intra-body communication at R-band frequencies," Sensors, vol. 18, no. 9 , p. $2752,2018$.

21. CST Microwave Studio, [Online]. Available: http://www.cst.com

22. C. Kissi; M. Särestöniemi; C. Pomalaza-Raez; M. Sonkki; and M. N. Srifi, "Low-UWB directive antenna for Wireless Capsule Endoscopy localization", BodyNets2018 conference, Oulu, Finland, October 2018.

23. IEEE Standard for Local and metropolitan area networks - Part 15.6: Wireless Body Area Networks." IEEE. 
24. T. Tuovinen, K. Yekeh Yazdandoost, J. Iinatti, "Comparison of the Performance of Two Different UWB Antennas for the use in WBAN On-Body Coimmunications," European Conference on Antennas and Propagation (EUCAP2012), 2012.

25. M. Särestöniemi, C. Pomalaza-Raez, M. Berg, C. Kissi, M. Hämäläinen. J. Iinatti, "In-Body Power Distribution for Abdominal Monitoring and Implant Communications Systems," ISWCS, September 2019

26. https://www.itis.ethz.ch/virtual-population/tissue-properties/database/dielectricproperties. 